\title{
A New and Self-Contained Proof of Borwein's Norm Duality Theorem
}

\author{
Francisco J. Aragón Artacho ${ }^{1}$
}

\begin{abstract}
Borwein's norm duality theorem establishes the equality between the outer (inner) norm of a sublinear mapping and the inner (outer) norm of its adjoint mappings. In this note we provide an extended version of this theorem with a new and self-contained proof relying only on the Hahn-Banach theorem. We also give examples showing that the assumptions of the theorem cannot be relaxed.
\end{abstract}

Key words: convex process, sublinear mapping, norm duality, inner norm, outer norm.

AMS 2000 Subject Classification: 49J53, 47H04, 54C60.

\footnotetext{
${ }^{1}$ Department of Statistics and Operations Research, University of Alicante, 03071 Alicante, Spain, francisco.aragon@ua.es. This author is supported by Grant BES-2003-0188 from FPI Program of MEC (Spain)
} 
Borwein's norm duality theorem was proved in [3] in a paper involving a general study of convex processes. A revised version of it was published later in [6] with more details and some applications. The proof of the theorem given in both papers is somewhat involved and relying on other results scattered in the literature, such as a Lagrange multiplier theorem given in [5, Theorem 3.1]. In view to the fundamental importance of this result in variational analysis and beyond, we feel that the availability of a self-contained and detailed proof would benefit everyone involved in research and teaching in this area. In this paper we provide such a proof simultaneously extending the original version of the theorem.

For mappings acting in Euclidean spaces, two different proofs are provided in [4, Theorem 5.4.10] and [10, Theorem 11.29]. The first one explicitly uses the finite dimensions. The second one is based on support functions and their properties. Because of the complexity of the proof and the use of a number of other results all stated in finite dimensions, the author is not in a position to determine whether the proof in [10] can be extended to infinitedimensional spaces. Here we present a proof in normed spaces the only prerequisite for which is the Hahn-Banach theorem. The proof follows partially the original Borwein's proof in [3].

Throughout the paper $X$ and $Y$ are real linear normed spaces whose norms are both denoted by $\|\cdot\|$. For a multivalued mapping acting from $X$ into the subsets of $Y$, denoted by $F: X \rightrightarrows Y$, we define its graph and its domain, respectively, by

$$
\operatorname{gph} F=\{(x, y) \in X \times Y \mid y \in F(x)\} \quad \text { and } \quad \operatorname{dom} F=\{x \in X \mid F(x) \neq \emptyset\},
$$

and the associated inverse mapping by

$$
F^{-1}(y)=\{x \in X \mid y \in F(x)\}, \quad \text { for } y \in Y
$$

A mapping $F: X \rightrightarrows Y$ is said to be positively homogeneous when $0 \in F(0)$ and $F(\lambda x)=$ $\lambda F(x)$ for all $\lambda>0$ and $x \in X$, or equivalently, when $\operatorname{gph} F$ is a cone in $X \times Y$. $F$ is said to be sublinear when it is positively homogeneous and, in addition, $F(x)+F\left(x^{\prime}\right) \subset F\left(x+x^{\prime}\right)$ for all $x, x^{\prime} \in X$; equivalently, when gph $F$ is a convex cone in $X \times Y$. A sublinear mapping whose domain is the whole space $X$ and which is single-valued on $X$ is a linear function. Sublinear mappings are introduced by R. T. Rockafellar in [8, 9] under the name convex processes.

For any sublinear mapping $F: X \rightrightarrows Y$, the outer norm $\|F\|^{+}$and the inner norm $\|F\|^{-}$ are defined by

$$
\|F\|^{+}=\sup _{x \in \mathbb{B}} \sup _{y \in F(x)}\|y\| \quad \text { and } \quad\|F\|^{-}=\sup _{x \in \mathbb{B}} \inf _{y \in F(x)}\|y\| .
$$

Here we follow the convention adopted in [10] setting $\inf _{y \in A}\|y\|=\infty$ and $\sup _{y \in A}\|y\|=-\infty$ if $A=\emptyset$. Another equivalent way to define these quantities (see [3] and [6]) is

$$
\|F\|^{+}=\inf \{r>0 \mid F(\mathbb{B}) \subset r \mathbb{B}\} \quad \text { and } \quad\|F\|^{-}=\inf \{r>0 \mid F(x) \cap r \mathbb{B} \neq \emptyset, \forall x \in \mathbb{B}\} .
$$

When $\operatorname{dom} F=X$ and $F$ is single-valued on $X$ both norms agree. For $F$ linear and bounded, both norms reduce to the operator norm $\|F\|$. Neither $\|F\|^{+}$nor $\|F\|^{-}$satisfy the conditions in the definition of a norm, since sublinear mappings do not form a vector space. 
Since the infimum over a nonempty set is greater or equal than the supremum, for any sublinear mapping with $\operatorname{dom} F=X$ one has $\|F\|^{+} \geq\|F\|^{-}$. But when $\operatorname{dom} F \neq X$, and thus $\|F\|^{-}=\infty$, we can have $\|F\|^{+}<\|F\|^{-}$. In fact, for a sublinear multivalued mapping, the inner and the outer norms cannot be finite simultaneously (see [1] for details).

Robinson gave in [7] a definition of the inner norm restricted to the domain, namely,

$$
\|F\|_{\mathrm{d}}^{-}=\sup _{x \in \mathbb{B} \cap \operatorname{dom} F} \inf _{y \in F(x)}\|y\| .
$$

This restriction to the domain changes significantly the inner norm so that the duality theorem does not hold anymore (for more, see [1]).

The norm duality theorem relates inner and outer norms of a mapping and its adjoint in the sense of Rockafellar [8]. Denoting $X^{*}$ and $Y^{*}$ the dual spaces of $X$ and $Y$, the upper adjoint of a positively homogeneous mapping $F: X \rightrightarrows Y$ is a mapping $F^{*+}: Y^{*} \rightrightarrows X^{*}$ defined as

$$
x^{*} \in F^{*+}\left(y^{*}\right) \Longleftrightarrow\left\langle x^{*}, x\right\rangle \leq\left\langle y^{*}, y\right\rangle \text { for all }(x, y) \in \operatorname{gph} F,
$$

while the lower adjoint is a mapping $F^{*-}: Y^{*} \rightrightarrows X^{*}$ having

$$
x^{*} \in F^{*-}\left(y^{*}\right) \Longleftrightarrow\left\langle x^{*}, x\right\rangle \geq\left\langle y^{*}, y\right\rangle \text { for all }(x, y) \in \operatorname{gph} F
$$

that is, gph $F^{*+}=-\operatorname{gph} F^{*-}$.

Following [10] we say that a mapping $F: X \rightrightarrows Y$ is inner semicontinuous at $\bar{x} \in \operatorname{dom} F$ if for every $y \in F(\bar{x})$ and every neighborhood $V$ of $y$ one can find a neighborhood $U$ of $\bar{x}$ with

$$
F(x) \cap V \neq \emptyset, \quad \text { for all } x \in U \text {. }
$$

The version of Borwein's theorem we prove is attached next, where, unlike in [3], we do not assume that $X$ is a Banach space.

Theorem 1 (Norm Duality). Let $F: X \rightrightarrows Y$ be a sublinear mapping between normed spaces $X$ and $Y$. Then

$$
\|F\|^{+}=\left\|F^{*-}\right\|^{-}=\left\|F^{*+}\right\|^{-} .
$$

Even more, if $Y$ is a Banach space and gph $F$ is closed, then

$$
\|F\|^{-}=\left\|F^{*-}\right\|^{+}=\left\|F^{*+}\right\|^{+} .
$$

In the proof we use the Hahn-Banach extension theorem (see, e.g., [11, Theorem 3.2]).

Theorem 2 (Hahn-Banach). Let $X$ be a real linear space, and let $p: X \rightarrow \mathbb{R}$ be a function such that $p(x+y) \leq p(x)+p(y)$ for all $x, y \in X$, and $p(\alpha x)=\alpha p(x)$ for all $x \in X$ and $\alpha \geq 0$. Let $M$ be a real linear subspace of $X$ and let $t: M \rightarrow \mathbb{R}$ be a linear function such that $t(x) \leq p(x)$ for all $x \in M$. Then there exists a linear function $T: X \rightarrow \mathbb{R}$ such that $T(x)=t(x)$ for all $x \in M$, and $T(x) \leq p(x)$ for all $x \in X$.

We also use the following corollary of the extension Theorem 2 (see, e.g., [2, Theorem 2.14]). 
Corollary 3 (Separation). Let $X$ be a real normed space and let $C \subset X$ be a nonempty closed convex set. If $x_{0} \notin C$, then there is $x^{*} \in X^{*}$ such that

$$
\left\langle x^{*}, x_{0}\right\rangle>\sup _{x \in C}\left\langle x^{*}, x\right\rangle .
$$

Proof of Theorem 1. First, note that $F^{*+}\left(y^{*}\right)=-F^{*-}\left(-y^{*}\right)$ for any $y^{*} \in Y^{*}$. Then

$$
\left\|F^{*-}\right\|^{-}=\left\|F^{*+}\right\|^{-} \quad \text { and } \quad\left\|F^{*-}\right\|^{+}=\left\|F^{*+}\right\|^{+} .
$$

By definition,

$$
\|F\|^{+}=\sup _{x \in \mathbb{B}} \sup _{y \in F(x)}\|y\|=\sup _{x \in \mathbb{B}} \sup _{y \in F(x)} \sup _{y^{*} \in \mathbb{B}}\left\langle y^{*}, y\right\rangle,
$$

and

$$
\left\|F^{*-}\right\|^{-}=\sup _{y^{*} \in \mathbb{B}} \inf _{x^{*} \in F^{*-}\left(y^{*}\right)}\left\|x^{*}\right\| .
$$

To prove the equality $\|F\|^{+}=\left\|F^{*-}\right\|^{-}$it is enough to show that

$$
\sup _{x \in \mathbb{B}} \sup _{y \in F(x)}\left\langle y^{*}, y\right\rangle=\inf _{x^{*} \in F^{*}-\left(y^{*}\right)}\left\|x^{*}\right\| \quad \text { for all } y^{*} \in \mathbb{B} .
$$

In fact, this holds true for any $y^{*} \in Y^{*}$. Fix $y^{*} \in Y^{*}$.

STEP 1. If $\inf _{x^{*} \in F^{*-}\left(y^{*}\right)}\left\|x^{*}\right\|<r$ for some $r>0$, then there exist $x^{*} \in F^{*-}\left(y^{*}\right)$ such that $\left\|x^{*}\right\|<r$. For any $\tilde{x} \in \mathbb{B}$ and $\tilde{y} \in F(\tilde{x})$ we have

$$
\left\langle y^{*}, \tilde{y}\right\rangle \leq\left\langle x^{*}, \tilde{x}\right\rangle \leq \sup _{x \in \mathbb{B}}\left\langle x^{*}, x\right\rangle=\left\|x^{*}\right\|<r,
$$

and then $\sup _{x \in \mathbb{B}} \sup _{y \in F(x)}\left\langle y^{*}, y\right\rangle \leq r$. Hence

$$
\sup _{x \in \mathbb{B}} \sup _{y \in F(x)}\left\langle y^{*}, y\right\rangle \leq \inf _{x^{*} \in F^{*-}\left(y^{*}\right)}\left\|x^{*}\right\| .
$$

SteP 2. To prove the inequality opposite to (3), assume that

$$
\sup _{x \in \mathbb{B}} \sup _{y \in F(x)}\left\langle y^{*}, y\right\rangle<r
$$

for some $r>0$. Pick $0<d<r$ such that

$$
\sup _{x \in \mathbb{B}} \sup _{y \in F(x)}\left\langle y^{*}, y\right\rangle \leq d .
$$

Define the mapping $H: X \rightrightarrows \mathbb{R}$ as

$$
H(x):=\left\{\left\langle y^{*}, y\right\rangle \mid y \in F(x+\mathbb{B})\right\}, \quad \text { for } x \in X .
$$


First, observe that gph $H$ is convex. Indeed, if $\left(x_{1}, z_{1}\right),\left(x_{2}, z_{2}\right) \in \operatorname{gph} H$ and $0<\lambda<1$, then there exist $y_{i} \in Y$ and $w_{i} \in \mathbb{B}$ with $z_{i}=\left\langle y^{*}, y_{i}\right\rangle$ and $y_{i} \in F\left(x_{i}+w_{i}\right)$, for $i=1,2$. Since $F$ is sublinear, we get

$$
\lambda y_{1}+(1-\lambda) y_{2} \in F\left(\lambda\left(x_{1}+w_{1}\right)+(1-\lambda)\left(x_{2}+w_{2}\right)\right) \subset F\left(\lambda x_{1}+(1-\lambda) x_{2}+\mathbb{B}\right),
$$

and thus,

$$
\lambda\left(x_{1}, z_{1}\right)+(1-\lambda)\left(x_{2}, z_{2}\right)=\left(\lambda x_{1}+(1-\lambda) x_{2},\left\langle y^{*}, \lambda y_{1}+(1-\lambda) y_{2}\right\rangle\right) \in \operatorname{gph} H
$$

We will show next that $H$ is inner semicontinuous at 0 . Take $\tilde{z} \in H(0)$ and $\varepsilon>0$. Let $\tilde{z}=\left\langle y^{*}, \tilde{y}\right\rangle$, for $\tilde{y} \in F(\tilde{w})$ and $\tilde{w} \in \mathbb{B}$. Since $\left\langle y^{*}, \cdot\right\rangle$ is continuous, there is some $\gamma>0$ such that $\left|\left\langle y^{*}, y\right\rangle-\tilde{z}\right| \leq \varepsilon$ when $\|y-\tilde{y}\| \leq \gamma$. Choose $\delta \in(0,1)$ such that $\delta\|\tilde{y}\| \leq \gamma$. If $\|x\| \leq \delta$, we have

$$
\|(1-\delta) \tilde{w}-x\| \leq\|(1-\delta) \tilde{w}\|+\|x\| \leq 1,
$$

and hence $(1-\delta) \tilde{w}-x \in \mathbb{B}$. Since $F$ is sublinear,

$$
(1-\delta) \tilde{y} \in F((1-\delta) \tilde{w})=F(x+((1-\delta) \tilde{w}-x)) \subset F(x+\mathbb{B}),
$$

whenever $\|x\| \leq \delta$. Moreover,

$$
\|(1-\delta) \tilde{y}-\tilde{y}\|=\delta\|\tilde{y}\| \leq \gamma
$$

and then

$$
\left|\left\langle y^{*},(1-\delta) \tilde{y}\right\rangle-\tilde{z}\right| \leq \varepsilon
$$

Therefore, for all $x \in \delta \mathbb{B}$, we have $\left\langle y^{*},(1-\delta) \tilde{y}\right\rangle \in H(x) \cap \mathbb{B}_{\varepsilon}(\tilde{z})$, and hence $H$ is inner semicontinuous at 0 as desired.

Let us now define a mapping $K: X \rightrightarrows \mathbb{R}$ by

$$
\operatorname{gph} K:=\operatorname{cone}(\operatorname{gph}(d-H)) \text {. }
$$

Clearly $K$ is a sublinear mapping. Since $H$ is inner semicontinuous at 0 , there is some neighborhood $U$ of 0 with $U \subset \operatorname{dom} H$, and therefore dom $K=X$. Consider

$$
k(x):=\inf \{z \mid z \in K(x)\} \quad \text { for } x \in X .
$$

Since $K$ is sublinear and $d-H(0) \subset \mathbb{R}_{+}$, we have

$$
K(x)+K(-x) \subset K(0) \subset \mathbb{R}_{+}
$$

This inclusion implies in particular that any point in $-K(-x)$ is a lower bound for the set of values $K(x)$, for any $x \in X$. Indeed, let $x \in X$ and $y \in-K(-x)$. Then (5) yields $K(x)-y \subset \mathbb{R}_{+}$, and thus

$$
y \leq z \text { for all } z \in K(x) .
$$

Therefore $k(x)$ is finite for all $x \in X$, and hence the function $k: X \rightarrow \mathbb{R}$ is well defined. Also, from the sublinearity of $K$ and the properties of the infimum, we have

$$
k(x+y) \leq k(x)+k(y) \quad \text { and } \quad k(\alpha x)=\alpha k(x), \quad \text { for all } x, y \in X \text { and } \alpha \geq 0 .
$$


Consider the subspace $M=\{0\} \subset X$ and define $t: M \rightarrow \mathbb{R}$ by $t(0):=k(0)=0$. Applying the Hahn-Banach extension Theorem 2 to $t$, we find a linear functional $T: X \rightarrow \mathbb{R}$ such that $T(0)=0$ and $T(x) \leq k(x)$ for all $x \in X$.

We will show now that $T$ is continuous at 0 and hence it is continuous on the whole $X$. Continuity at 0 means that for all $\varepsilon>0$ there is $\delta>0$ such that $\left(T(x)+\mathbb{R}_{+}\right) \cap \varepsilon \mathbb{B} \neq \emptyset$, whenever $x \in \delta \mathbb{B}$. Let $z \in d-H(0)$. Take $0<\lambda<1$ and a neighborhood $V$ of $z$ such that $\lambda V \subset \varepsilon \mathbb{B}$. Since $H$ is inner semicontinuous at 0 , there is some $\delta>0$ such that

$$
(d-H(x)) \cap V \neq \emptyset, \quad \text { for all } x \in(\delta / \lambda) \mathbb{B} .
$$

Since $d-H(x) \subset k(x)+\mathbb{R}_{+}$and $k(x) \geq T(x)$, we have $d-H(x) \subset T(x)+\mathbb{R}_{+}$,

$$
\left(T(x)+\mathbb{R}_{+}\right) \cap V \neq \emptyset, \quad \text { for all } x \in(\delta / \lambda) \mathbb{B},
$$

and then

$$
\left(T(\lambda x)+\mathbb{R}_{+}\right) \cap \lambda V \neq \emptyset, \quad \text { for all } x \in(\delta / \lambda) \mathbb{B}
$$

which yields

$$
\left(T(x)+\mathbb{R}_{+}\right) \cap \varepsilon \mathbb{B} \neq \emptyset, \quad \text { for all } x \in \delta \mathbb{B} .
$$

This means that for all $x \in \delta \mathbb{B}$ there exists some $z \geq T(x)$ with $|z| \leq \varepsilon$. Since $T$ is linear, $T(-x)=-T(x)$, and therefore $|T(x)| \leq \varepsilon$ for all $x \in \delta \mathbb{B}$. This shows the continuity of $T$.

The inclusion $d-H(x)-T(x) \subset \mathbb{R}_{+}$is equivalent to

$$
d-\left\langle y^{*}, y\right\rangle-T(x) \geq 0, \quad \text { whenever } x \in X \text { and } y \in F(x+\mathbb{B}) .
$$

Let $x^{*} \in X^{*}$ be such that $\left\langle x^{*}, x\right\rangle=-T(x)$ for all $x \in X$. Then

$$
\left\langle y^{*}, y\right\rangle-\left\langle x^{*}, x\right\rangle \leq d, \quad \text { for all } x \in X \text { and } y \in F(x+\mathbb{B}) \text {. }
$$

Pick $y \in F(x)$ and $\lambda>0$. Then $\lambda y \in F(\lambda x)$ and

$$
\left\langle y^{*}, \lambda y\right\rangle-\left\langle x^{*}, \lambda x\right\rangle \leq d
$$

or equivalently,

$$
\left\langle y^{*}, y\right\rangle-\left\langle x^{*}, x\right\rangle \leq d / \lambda .
$$

Passing to the limit with $\lambda \rightarrow \infty$, we obtain $x^{*} \in F^{*-}\left(y^{*}\right)$. Let now $x \in \mathbb{B}$. Since $0 \in F(0)$, we have $0 \in F(-x+\mathbb{B})$, and hence

$$
\left\langle y^{*}, 0\right\rangle-\left\langle x^{*},-x\right\rangle \leq d
$$

Therefore $\left\|x^{*}\right\| \leq d<r$ and then $\inf _{x^{*} \in F^{*-}\left(y^{*}\right)}\left\|x^{*}\right\|<r$. This completes Step 2 and the proof of (1).

We will complete the proof of (2) by showing that $\|F\|^{-}=\left\|F^{*+}\right\|^{+}$when $Y$ is a Banach space and gph $F$ is closed. 
SteP 3. Let $\|F\|^{-}<r$ for some $r>0$. Then for any $\tilde{x} \in \mathbb{B}$ there is some $\tilde{y} \in F(\tilde{x})$ with $\|\tilde{y}\|<r$. Given some $y^{*} \in \mathbb{B}$ and $x^{*} \in F^{*+}\left(y^{*}\right)$ we have

$$
\left\langle x^{*}, \tilde{x}\right\rangle \leq\left\langle y^{*}, \tilde{y}\right\rangle \leq\|\tilde{y}\|<r .
$$

Since this last inequality is valid for any $\tilde{x} \in \mathbb{B}$, we obtain $\left\|x^{*}\right\| \leq r$, and therefore $\left\|F^{*+}\right\|^{+} \leq$ $r$. Hence

$$
\|F\|^{-} \geq\left\|F^{*+}\right\|^{+}
$$

SteP 4. To prove $\|F\|^{-} \leq\left\|F^{*+}\right\|^{+}$, suppose that $\left\|F^{*+}\right\|^{+}<r$. Pick $s>0$ with

$$
\sup _{x^{*} \in F^{*+}(\mathbb{B})}\left\|x^{*}\right\|=\left\|F^{*+}\right\|^{+} \leq s<r .
$$

Thus, $F^{*+}(\mathbb{B}) \subset s \mathbb{B}$. We will show that $\left(F^{-1}(\mathbb{B})\right)^{\circ} \subset F^{*+}(\mathbb{B})$, and hence

$$
\left(F^{-1}(\mathbb{B})\right)^{\circ} \subset s \mathbb{B} \text {. }
$$

Let $x^{*} \in\left(F^{-1}(\mathbb{B})\right)^{\circ}$. Then

$$
\left\langle x^{*}, x\right\rangle \leq 1 \quad \text { for all } x \in F^{-1}(\mathbb{B})
$$

or, equivalently,

$$
\sup _{y \in \mathbb{B}} \sup _{x \in F^{-1}(\mathbb{B})}\left\langle x^{*}, x\right\rangle \leq 1 .
$$

This latter inequality is analogous to (4), with $d=1$ and $F$ replaced by $F^{-1}$ and thus, with $y$ and $y^{*}$ replaced by $x$ and $x^{*}$, respectively. By repeating the argument in the proof of Step 2, we find some $y^{*} \in\left(F^{-1}\right)^{*-}\left(x^{*}\right)=\left(F^{*+}\right)^{-1}\left(x^{*}\right)$ with $\left\|y^{*}\right\| \leq 1$. But then $x^{*} \in F^{*+}(\mathbb{B})$ and (6) follows.

Now we will show that (6) implies

$$
s^{-1} \mathbb{B} \subset \operatorname{cl} F^{-1}(\mathbb{B}) .
$$

Indeed, if $x \notin \mathrm{cl} F^{-1}(\mathbb{B})$, then from the separation Corollary 3 , there exists $\tilde{x}^{*} \in X^{*}$ with

$$
\left\langle\tilde{x}^{*}, x\right\rangle>\sup _{z \in \operatorname{cl} F^{-1}(\mathbb{B})}\left\langle\tilde{x}^{*}, z\right\rangle \geq\left\langle\tilde{x}^{*}, 0\right\rangle=0 .
$$

Let $\lambda>0$ be such that

$$
\sup _{z \in \operatorname{cl} F^{-1}(\mathbb{B})}\left\langle\tilde{x}^{*}, z\right\rangle<\lambda^{-1}<\left\langle\tilde{x}^{*}, x\right\rangle .
$$

Then we have

$$
\left\langle\lambda \tilde{x}^{*}, x\right\rangle>1>\sup _{z \in \operatorname{cl} F^{-1}(\mathbb{B})}\left\langle\lambda \tilde{x}^{*}, z\right\rangle .
$$

In particular, this implies that $\lambda \tilde{x}^{*} \in\left(F^{-1}(\mathbb{B})\right)^{\circ} \subset s \mathbb{B}$. Hence,

$$
s \geq\left\|\lambda \tilde{x}^{*}\right\| \geq\left\langle\lambda \tilde{x}^{*}, x /\|x\|\right\rangle>\frac{1}{\|x\|},
$$


and therefore $x \notin s^{-1} \mathbb{B}$. Thus, (6) holds.

We will show next that

$$
\text { int } s^{-1} \mathbb{B} \subset \operatorname{int} F^{-1}(\mathbb{B}) \text {. }
$$

Let $x \in \operatorname{int} s^{-1} \mathbb{B}$. Then from (6) we have $x \in \operatorname{int} \operatorname{cl}^{-1}(\mathbb{B})$, and hence, there is some $\varepsilon>0$ such that

$$
\mathbb{B}_{\varepsilon}(x) \subset \operatorname{cl} F^{-1}(\mathbb{B}) .
$$

Since $\operatorname{cl} F^{-1}(\mathbb{B}) \subset F^{-1}(\mathbb{B})+(\varepsilon / 2) \mathbb{B}$, we have

$$
x+\varepsilon \mathbb{B} \subset F^{-1}(\mathbb{B})+\frac{\varepsilon}{2} \mathbb{B} .
$$

Multiplying the last inclusion by $1 / 2$ and adding $x / 2$ to both sides we obtain

$$
\mathbb{B}_{\varepsilon / 2}(x) \subset \frac{1}{2} F^{-1}(\mathbb{B})+\frac{1}{2} \mathbb{B}_{\varepsilon / 2}(x) .
$$

We will show next that $\mathbb{B}_{\varepsilon / 2}(x) \subset F^{-1}(\mathbb{B})$. From (9) we have

$$
\mathbb{B}_{\varepsilon / 2}(x) \subset \frac{1}{2} F^{-1}(\mathbb{B})+\frac{1}{4} F^{-1}(\mathbb{B})+\ldots+\frac{1}{2^{k}} F^{-1}(\mathbb{B})+\frac{1}{2^{k+1}} \mathbb{B}_{\varepsilon / 2}(x),
$$

for all $k \in \mathbb{N}$. Let $z \in \mathbb{B}_{\varepsilon / 2}(x)$. Then, there is $z_{1}, \ldots, z_{k} \in F^{-1}(\mathbb{B})$ such that

$$
z \in \frac{1}{2} z_{1}+\frac{1}{4} z_{2}+\ldots+\frac{1}{2^{k}} z_{k}+\frac{1}{2^{k+1}} \mathbb{B}_{\varepsilon / 2}(x) .
$$

Hence, by induction, for every $n \in \mathbb{N}$, there is $z_{n} \in F^{-1}(\mathbb{B})$ such that $z=\sum_{n \in \mathbb{N}} 2^{-n} z_{n}$. Thus, for each $n \in \mathbb{N}$, there is $y_{n} \in \mathbb{B}$ with $\left(z_{n}, y_{n}\right) \in \operatorname{gph} F$. Consider the sequences

$$
\tilde{z}_{n}:=\sum_{\kappa=1}^{n} \frac{1}{2^{k}} z_{k} \quad \text { and } \quad \tilde{y}_{n}:=\sum_{\kappa=1}^{n} \frac{1}{2^{k}} y_{k},
$$

for $n \in \mathbb{N}$. Clearly, $\tilde{z}_{n}$ converges to $z$. Moreover, since

$$
\left\|\tilde{y}_{n}-\tilde{y}_{n-1}\right\|=\frac{1}{2^{n}}\left\|y_{n}\right\| \leq \frac{1}{2^{n}}, \quad \text { for all } n \in \mathbb{N},
$$

we conclude that $\tilde{y}_{n}$ is a Cauchy sequence in $Y$. Then, since $Y$ is a Banach space, it converges to some $y \in Y$ whose norm satisfies

$$
\|y\| \leq \sum_{n \in \mathbb{N}} \frac{1}{2^{n}}\left\|y_{n}\right\| \leq 1 .
$$

Therefore, the sequence $\left(\tilde{z}_{n}, \tilde{y}_{n}\right)$ converges to $(z, y)$, and because of the closedness of gph $F$, we have $z \in F^{-1}(y) \subset F^{-1}(\mathbb{B})$. 
Summarizing, we have shown that for any $x \in \operatorname{int} s^{-1} \mathbb{B}$ there exists some $\varepsilon>0$ such that $\mathbb{B}_{\varepsilon / 2}(x) \subset F^{-1}(\mathbb{B})$. This yields

$$
\text { int } s^{-1} \mathbb{B} \subset \operatorname{int} F^{-1}(\mathbb{B}) \text {. }
$$

Then

$$
r^{-1} \mathbb{B} \subset \text { int } s^{-1} \mathbb{B} \subset F^{-1}(\mathbb{B}),
$$

and thus, $\|F\|^{-} \leq r$. This completes the proof of Step 4 and hence we have shown that $\|F\|^{-}=\left\|F^{*+}\right\|^{+}$, concluding the proof of (2).

Given a sublinear mapping $F: X \rightrightarrows Y$, where $X$ is a normed space and $Y$ is a Banach space, we can define a sublinear mapping $\bar{F}: X \rightrightarrows Y$ by gph $\bar{F}:=\operatorname{clgph} F$, where $\operatorname{cl} A$ denotes the closure of the set $A$. Since gph $F \subset \operatorname{gph} \bar{F}$, it is clear that $\operatorname{gph} \bar{F}^{*+} \subset \operatorname{gph} F^{*+}$. On the other hand, suppose that $\left(y^{*}, x^{*}\right) \in \operatorname{gph} F^{*+}$. Let $(x, y) \in \operatorname{gph} \bar{F}$. Then there is a sequence $\left(x_{n}, y_{n}\right) \in \operatorname{gph} F$ with $\left(x_{n}, y_{n}\right) \rightarrow(x, y)$, and hence $\left\langle x^{*}, x_{n}\right\rangle \leq\left\langle y^{*}, y_{n}\right\rangle$. Taking limits, we have $\left\langle x^{*}, x\right\rangle \leq\left\langle y^{*}, y\right\rangle$, and since this is true for all $(x, y) \in \operatorname{gph} \bar{F}$, we obtain $\left(y^{*}, x^{*}\right) \in \operatorname{gph} \bar{F}^{*+}$. Therefore

$$
\bar{F}^{*+}=F^{*+} .
$$

Also, given $x \in X$, if $y \in F(x)$, then $(x, y) \in \operatorname{gph} F \subset \operatorname{gph} \bar{F}$, and hence $y \in \bar{F}(x)$. Thus, applying (10) and Theorem 1,

$$
\|F\|^{-}=\sup _{x \in \mathbb{B}} \inf _{y \in F(x)}\|y\| \geq \sup _{x \in \mathbb{B}} \inf _{y \in \bar{F}(x)}\|y\|=\|\bar{F}\|^{-}=\left\|\bar{F}^{*+}\right\|^{+}=\left\|F^{*+}\right\|^{+} .
$$

In fact, this inequality follows directly from Step 3 of Theorem 1, where $Y$ does not need to be a Banach space.

Corollary 4. Let $F: X \rightrightarrows Y$ be a sublinear mapping between normed spaces $X$ and $Y$. Then

$$
\|F\|^{-} \geq\left\|F^{*+}\right\|^{+}=\left\|F^{*-}\right\|^{+} .
$$

The natural question now is the following: is the equality (2) valid without the closedness assumption? The answer is no, even for a single-valued mapping acting between Banach spaces, as the following example shows.

Consider the Banach space $\ell_{1}$ which elements are the scalar-valued sequences $x=\left\{x_{n}\right\}_{n \in \mathbb{N}}$ satisfying $\sum_{n \in \mathbb{N}}\left|x_{n}\right|<\infty$, endowed with the norm

$$
\|x\|_{1}=\sum_{n \in \mathbb{N}}\left|x_{n}\right| .
$$

Let $F: \ell_{1} \rightarrow \ell_{1}$ defined by

$$
F(x)= \begin{cases}x & \text { if } x_{n} \neq 0 \text { for a finite number of } n \\ \emptyset & \text { otherwise }\end{cases}
$$


for $x=\left\{x_{n}\right\}_{n \in \mathbb{N}}$. It is easy to check that $F$ is a sublinear mapping having $\operatorname{dom} F \neq X$ (e.g., for $x=\left\{1 / 2^{n}\right\}_{n \in \mathbb{N}} \in \ell_{1}$ we have $F(x)=\emptyset$ ). Thus $\|F\|^{-}=\infty$. On the other hand, given $x=\left\{x_{n}\right\}_{n \in \mathbb{N}} \in \ell_{1}$, we can define $x^{k}=\left\{x_{n}^{k}\right\}_{n \in \mathbb{N}} \in \ell_{1}$ by

$$
x_{n}^{k}:= \begin{cases}x_{n} & \text { for } n \leq k, \\ 0 & \text { for } n>k .\end{cases}
$$

Then

$$
\lim _{k \rightarrow \infty}\left\|x-x^{k}\right\|=\lim _{k \rightarrow \infty}\left(\sum_{n=k+1}^{\infty}\left|x_{n}\right|\right)=\lim _{k \rightarrow \infty}\left(\sum_{n \in \mathbb{N}}\left|x_{n}\right|-\sum_{n=1}^{k}\left|x_{n}\right|\right)=0,
$$

and thus $x^{k} \rightarrow x$. Since $F\left(x^{k}\right)=x^{k}$ for all $k \in \mathbb{N}$, we have $\bar{F}(x)=x$ for all $x \in \ell_{1}$. Then $\left\|F^{*+}\right\|^{+}=\left\|\bar{F}^{*+}\right\|^{+}=\|\bar{F}\|^{-}=1$, but $\|F\|^{-}=\infty$.

A modification of this example serve us to show that the first equality in (2) is not valid in general when $Y$ is not complete. Consider the Banach space $c_{0}$ whose elements are the scalar-valued sequences $x=\left\{x_{n}\right\}_{n \in \mathbb{N}}$ satisfying $\lim _{n \rightarrow \infty} x_{n}=0$, endowed with the norm

$$
\|x\|_{\infty}=\sup _{n \in \mathbb{N}}\left|x_{n}\right| .
$$

Let $c_{00}$ be the subspace of $c_{0}$ consisting of all the sequences with a finite number of nonzero elements. It is well known that $c_{00}$ is a normed space which is not complete. Consider the mapping $F: c_{0} \rightarrow c_{00}$ defined by

$$
F(x)= \begin{cases}x & \text { if } x \in c_{00}, \\ \emptyset & \text { otherwise }\end{cases}
$$

for $x=\left\{x_{n}\right\}_{n \in \mathbb{N}}$. It is not difficult to check that $F$ is a sublinear mapping with $\operatorname{dom} F \neq c_{0}$, and hence $\|F\|^{-}=\infty$. In this case gph $F$ is closed, but $\left\|F^{*+}\right\|^{+}=1$. Indeed, given $\left(y^{*}, x^{*}\right) \in\left(c_{00}^{*}, c_{0}^{*}\right)$, we have

$$
\left(y^{*}, x^{*}\right) \in \operatorname{gph} F^{*+} \Longleftrightarrow\left\langle x^{*}, x\right\rangle=\left\langle y^{*}, x\right\rangle \quad \text { for all } x \in c_{00} .
$$

Fix $y^{*} \in \mathbb{B}$ and $x^{*} \in F^{*+}\left(y^{*}\right)$. Then

$$
\left\|x^{*}\right\|=\sup _{x \in c_{0} \cap \mathbb{B}}\left\langle x^{*}, x\right\rangle \geq \sup _{x \in c_{00} \cap \mathbb{B}}\left\langle x^{*}, x\right\rangle=\sup _{x \in c_{0} \cap \mathbb{B}}\left\langle y^{*}, x\right\rangle=\left\|y^{*}\right\| .
$$

Moreover, we must have $\left\|x^{*}\right\|=\left\|y^{*}\right\|$. Otherwise, there is some $\varepsilon>0$ such that $\left\|x^{*}\right\|>$ $\left\|y^{*}\right\|+\varepsilon$, and thus, there exists some $\tilde{x} \in c_{0} \cap \mathbb{B}$ with

$$
\left\langle x^{*}, \tilde{x}\right\rangle>\left\|y^{*}\right\|+\varepsilon=\sup _{y \in c_{0} \cap \mathbb{B}}\left\langle y^{*}, y\right\rangle+\varepsilon .
$$

This implies

$$
\left\langle x^{*}, \tilde{x}\right\rangle>\left\langle y^{*}, y\right\rangle+\varepsilon=\left\langle x^{*}, y\right\rangle+\varepsilon \text { for any } y \in c_{00} \cap \mathbb{B} \text {. }
$$


If $\tilde{x}=\left\{\tilde{x}_{n}\right\}_{n \in \mathbb{N}}$, consider the sequence $z^{n}:=\left\{\tilde{x}_{1}, \ldots, \tilde{x}_{n}, 0, \ldots\right\} \in c_{00} \cap \mathbb{B}$, for $n \in \mathbb{N}$. Since

$$
\left\|z^{n}-\tilde{x}\right\|_{\infty}=\sup _{k>n}\left|\tilde{x}_{k}\right|
$$

and $\tilde{x} \in c_{0}$, we have that $z^{n}$ converges to $\tilde{x}$. But (13) implies

$$
\left\langle x^{*}, \tilde{x}\right\rangle>\left\langle x^{*}, z^{n}\right\rangle+\varepsilon \quad \text { for all } n \in \mathbb{N} \text {. }
$$

Then, passing to the limit with $n \rightarrow \infty$, we obtain a contradiction. Hence, we have $\left\|x^{*}\right\|=$ $\left\|y^{*}\right\| \leq 1$, and therefore $\left\|F^{*+}\right\|^{+}=1 \neq\|F\|^{-}=\infty$.

Now the next question would be whether the closedness condition is necessary in order to have (2). The answer turns out to be again negative. Indeed, let $F: \mathbb{R} \rightarrow \mathbb{R}$ be defined by

$$
F(x)= \begin{cases}(x, \infty) & \text { for } x \neq 0 \\ {[0, \infty)} & \text { for } x=0\end{cases}
$$

The graph of this mapping is not closed, but it has $\|F\|^{-}=\left\|F^{*+}\right\|^{+}=\left\|F^{*-}\right\|^{+}=1$.

In [3] Borwein considered another sufficient condition for having the equality (2): the inner semicontinuity of the mapping $F$ (there called lower semicontinuity). As we can see from the following example, neither the closedness nor the inner semicontinuity conditions are necessary for having (2).

Let $F: \mathbb{R} \rightarrow \mathbb{R}$ be a sublinear mapping defined by

$$
F(x)= \begin{cases}(0, \infty) & \text { for } x>0 \\ 0 & \text { for } x=0 \\ \emptyset & \text { for } x<0\end{cases}
$$

Here $F$ does not have the graph closed and it is not inner semicontinuous at 0 , but it has $\|F\|^{-}=\left\|F^{*+}\right\|^{+}=\infty$.

These examples leads us to the next result.

Corollary 5. Let $F: X \rightrightarrows Y$ be a sublinear mapping between normed spaces $X$ and $Y$. Let $\bar{F}$ be the closed sublinear mapping defined by gph $\bar{F}=\operatorname{clgph} F$. If $\operatorname{dom} \bar{F} \neq X$ or $F$ has the property

$$
\bar{F}(x)=\operatorname{cl} F(x) \text { for all } x \neq 0,
$$

then

$$
\|F\|^{-}=\left\|F^{*+}\right\|^{+}=\left\|F^{*-}\right\|^{+} .
$$


Proof. Because of (11), we just have to prove that $\|F\|^{-}=\|\bar{F}\|^{-}$. If $\operatorname{dom} \bar{F} \neq X$, then

$$
\|F\|^{-} \geq\|\bar{F}\|^{-}=\infty
$$

and thus we obtain (15). Now assume that $F$ verifies (14). We will show that

$$
\inf _{y \in F(x)}\|y\|=\inf _{y \in \bar{F}(x)}\|y\|, \quad \text { for all } x \in X .
$$

For $x=0$ this is true, since $(0,0) \in \operatorname{gph} F$. Consider now $x \in X \backslash\{0\}$. Because of (14), proving (16) is equivalent to show that

$$
\inf _{y \in F(x)}\|y\|=\inf _{y \in \operatorname{cl} F(x)}\|y\|,
$$

which is always valid. Taking the relevant suprema we complete the proof.

We will now show that the property (14) is weaker than both closedness and inner semicontinuity. Therefore, replacing the second part of Theorem 1 with the statement of Corollary 5 gives us a true generalization of Borwein's theorem. Indeed, if $F$ is a sublinear mapping with closed graph it is clear that $\bar{F}=F$ and $\operatorname{cl} F(x)=F(x)$ for all $x \in X$. This implies (14). Now suppose that $F$ is inner semicontinuous at 0 . Since always $\operatorname{cl} F(x) \subset \bar{F}(x)$ for any $x \in X$, we just need to prove the opposite inclusion. Let $y \in \bar{F}(x)$, for some $x \in X$. Then there is a sequence $\left(x_{n}, y_{n}\right) \in \operatorname{gph} F$ such that $\left(x_{n}, y_{n}\right) \rightarrow(x, y)$. Let $V_{k}:=(1 / k) \mathbb{B}$ for $k \in \mathbb{N}$. Since $F$ is inner semicontinuous at 0 and $0 \in F(0)$, for every $k \in \mathbb{N}$ there is a neighborhood $U_{k}$ of 0 such that

$$
F(x) \cap V_{k} \neq \emptyset, \quad \text { for any } x \in U_{k} \text {. }
$$

Since $x-x_{n} \rightarrow 0$, for every $k \in \mathbb{N}$ there is some $n_{k} \geq k$ with $x-x_{n_{k}} \in U_{k}$. Then from (17) there is some $w_{k} \in(1 / k) \mathbb{B} \cap F\left(x-x_{n_{k}}\right)$, for every $k \in \mathbb{N}$. Hence $w_{k} \rightarrow 0$ and $\left(x-x_{n_{k}}, w_{k}\right) \in \operatorname{gph} F$, and therefore,

$$
\left(x, y_{n_{k}}+w_{k}\right)=\left(x_{n_{k}}, y_{n_{k}}\right)+\left(x-x_{n_{k}}, w_{k}\right) \in \operatorname{gph} F,
$$

having $y_{n_{k}}+w_{k} \stackrel{k}{\longrightarrow} y$. Thus $y \in \operatorname{cl} F(x)$ and this implies (14).

Acknowledgement. I would like to thank Prof. A. L. Dontchev for his helpful comments and suggestions on the manuscript. 


\section{References}

[1] F. J. Aragón, A. L. Dontchev, On the Inner and Outer Norms of Sublinear Mappings, Set-Valued Analysis, DOI 10.1007/s11228-006-0034-4.

[2] J.F. Bonnans and A. Shapiro, Perturbation Analysis of Optimization Problems, Springer-Verlag, New York, 2000.

[3] J. M. Borwein, Adjoint Process Duality, Math. Oper. Res. 8 (1983), no. 3, 403-434.

[4] J. M. Borwein and A. S. Lewis, Convex Analysis and Nonlinear Optimization, SpringerVerlag, New York, 2000.

[5] J. M. Borwein, A Lagrange Multiplier Theorem and a Sandwich Theorem for Convex Relations, Math. Scand. 48 (1981), 189-204.

[6] J. M. Borwein, Norm Duality for Convex Processes and Applications. J. Optim. Theory Appl. 48 (1986), no. 1, 53-64.

[7] S. M. Robinson, Normed Convex Processes, Trans. Amer. Math. Soc. 174 (1972), $127-140$.

[8] R. T. Rockafellar, Convex Analysis, Princeton University Press, Princeton, N.J. 1970.

[9] R. T. Rockafellar, Monotone Processes of Convex and Concave Type, Memoirs of the American Mathematical Society 77, Providence, R.I. 1967.

[10] R. T. Rockafellar, R. J.-B. Wets, Variational Analysis, Springer-Verlag, Berlin, 1998.

[11] W. Rudin, Functional Analysis, McGraw-Hill, New York, 1973. 